\title{
A study of ocular sarcoidosis using international workshop for ocular sarcoidosis criteria in a south Indian population
}

\author{
Yogish S. Kamath ${ }^{1, *}$, Surabhi Abbagani ${ }^{2}$ \\ ${ }^{\mathbf{1}}$ Associate Professor, ${ }^{2}$ Junior Resident, Dept. of Ophthalmology, Kasturba Medical College, Manipal (Manipal Academy of \\ Higher Education), Karnataka, India
}

*Corresponding Author:

Email: dryogishkamath@yahoo.co.in

\begin{abstract}
Purpose: To study the clinical profile of patients with ocular sarcoidosis using the new International Workshop for Ocular Sarcoidosis (IWOS) guidelines, in a tertiary care hospital in South India.

Materials and Methods: A retrospective observational study where the records of patients diagnosed to have sarcoidosis were analyzed and regraded according to the new guidelines by the International Workshop for Ocular Sarcoidosis.

Results: Among the 36 sarcoidosis patient records evaluated in the study period, respiratory involvement was found in $80.6 \%$ whereas ocular involvement in $38.9 \%$ cases. Uveitis was the most common ocular manifestation seen in $59.1 \%$ of those who underwent ocular evaluation. Applying the IWOS guidelines in our patients with uveitis, we found, two had definite ocular sarcoidosis; seven had presumed ocular sarcoidosis; four had probable ocular sarcoidosis and none with possible ocular sarcoidosis. Conclusion: Sarcoidosis is not uncommon in India. Ocular features are common and need to be screened in all patients diagnosed with systemic sarcoidosis. An ophthalmologist can use IWOS criteria in cases of uveitis to clinically diagnose and refer cases for systemic evaluation of sarcoidosis.
\end{abstract}

Keywords: Angiotensin converting enzyme, Sarcoidosis, Uveitis.

\section{Introduction}

Sarcoidosis is a chronic granulomatous disease involving multiple systems including the eye. ${ }^{1}$ Ocular sarcoidosis usually presents as a chronic granulomatous uveitis involving both eyes. ${ }^{2}$ However, it is often overlooked in favor of tuberculosis, which can also present with similar clinical features. Especially in regions endemic for tuberculosis. ${ }^{3}$ There is a wide difference in the line of subsequent management of the two conditions.

The international workshop on ocular sarcoidosis (IWOS) laid down certain diagnostic criteria to aid the identification of ocular sarcoidosis. ${ }^{4}$ Our study aimed to apply these revised criteria to patients presenting to our center with a diagnosis of sarcoidosis, to assess ocular involvement.

\section{Materials and Methods}

The study was conducted in the Department of Ophthalmology, at a multi-specialty tertiary care University Hospital in South India. The study was conducted according to the guidelines of the declaration of Helsinki and had received the Institutional ethics committee approval. The records of all patients diagnosed with sarcoidosis by the Pulmonologist or Physician, in whom tuberculosis had been excluded by means of a Mantoux test and/or chest x-ray, were analyzed. The period of study was restricted from 2010 to 2016 due to the availability of records. The ocular clinical features were recorded and re-graded as per the guidelines of the IWOS, and results noted. The clinical features recorded included a detailed ocular and systemic history, slit lamp biomicroscopic anterior segment evaluation, fundus evaluation with indirect ophthalmoscopy. Systemic evaluation included a general dermatological evaluation for any papulonodular lesions, chest x-ray or CT scan for hilar lymphadenopathy, serum angiotensin converting enzyme (ACE) levels, Bronchoalveolar lavage (BAL), biopsy of skin lesions or lymph nodes. The results of the systemic examination and investigations performed by other specialties including dermatology, pulmonology and internal medicine were also recorded.

\section{Results}

A total of 36 patients had been diagnosed with sarcoidosis according to the hospital database.

$22(61.1 \%)$ of these patients had undergone an ophthalmological evaluation, among whom $13(59 \%)$ were females and $9(41 \%)$ were males.

Table 1: Systemic involvement

\begin{tabular}{|l|c|c|}
\hline System involved & $\begin{array}{c}\text { Number of } \\
\text { patients }\end{array}$ & Percentage \\
\hline Respiratory & 29 & $80.6 \%$ \\
\hline Ocular & 14 & $38.9 \%$ \\
\hline Dermatological & 8 & $22.2 \%$ \\
\hline Neurological & 4 & $11.1 \%$ \\
\hline
\end{tabular}

Table 2: Ocular involvement

\begin{tabular}{|l|c|c|}
\hline \multicolumn{1}{|c|}{ Ocular feature } & $\begin{array}{c}\text { Number of } \\
\text { patients }\end{array}$ & Percentage \\
\hline Uveitis & 13 & $59.1 \%$ \\
\hline $3^{\text {rd }}$ cranial nerve palsy & 1 & $4.5 \%$ \\
\hline $\begin{array}{l}\text { Other ocular } \\
\text { conditions }\end{array}$ & 2 & $9 \%$ \\
\hline
\end{tabular}




\begin{tabular}{|l|c|c|}
\hline $\begin{array}{l}\text { Primary open angle } \\
\text { glaucoma; refractive } \\
\text { error) }\end{array}$ & & \\
\hline Normal ocular study & 6 & $27.4 \%$ \\
\hline
\end{tabular}

Table 3: Anterior segment manifestations in patients with uveitis (Total number: 13 patients)

\begin{tabular}{|l|c|c|}
\hline \multicolumn{1}{|c|}{ Clinical Sign } & $\begin{array}{c}\text { Number of } \\
\text { patients }\end{array}$ & Percentage \\
\hline Conjunctival nodule & 1 & $7.7 \%$ \\
\hline $\begin{array}{l}\text { Large keratic } \\
\text { precipitates }\end{array}$ & 11 & $84.61 \%$ \\
\hline Iris nodules & 10 & $76.92 \%$ \\
\hline $\begin{array}{l}\text { Peripheral anterior } \\
\text { synechiae }\end{array}$ & 3 & $23.07 \%$ \\
\hline $\begin{array}{l}\text { Posterior synechiae } \\
\text { (broad based) }\end{array}$ & 11 & $84.61 \%$ \\
\hline Cataract & 5 & $38.46 \%$ \\
\hline
\end{tabular}

Table 4: Posterior segment manifestations in patients with uveitis (total number: 13 patients)

\begin{tabular}{|l|c|c|}
\hline Clinical sign & $\begin{array}{c}\text { Number of } \\
\text { patients }\end{array}$ & Percentage \\
\hline Vitritis & 11 & $84.61 \%$ \\
\hline Choroiditis & 3 & $23.07 \%$ \\
\hline $\begin{array}{l}\text { Retinal } \\
\text { vasculitis }\end{array}$ & 4 & $30.76 \%$ \\
\hline Disc edema & 1 & $7.7 \%$ \\
\hline
\end{tabular}

Table 5. Investigations performed for sarcoidosis (Total number: 36 patients)

\begin{tabular}{|l|c|c|}
\hline \multicolumn{1}{|c|}{ Investigation } & $\begin{array}{c}\text { Number of patients with positive results/ } \\
\text { number of patients in whom test was } \\
\text { performed }\end{array}$ & Percentage \\
\hline $\begin{array}{l}\text { Chest X-ray/CTscan for Hilar } \\
\text { lymphadenopathy }\end{array}$ & $32 / 36$ & $88.9 \%$ \\
\hline $\begin{array}{l}\text { Serum Angiotensin converting } \\
\text { enzyme }\end{array}$ & $25 / 32$ & $78.1 \%$ \\
\hline $\begin{array}{l}\text { Skin biopsy/BAL positivity for } \\
\text { sarcoidosis }\end{array}$ & $4 / 4$ & $100 \%$ \\
\hline Negative Mantoux test & $18 / 18$ & $100 \%$ \\
\hline
\end{tabular}

Chart 1: Age distribution

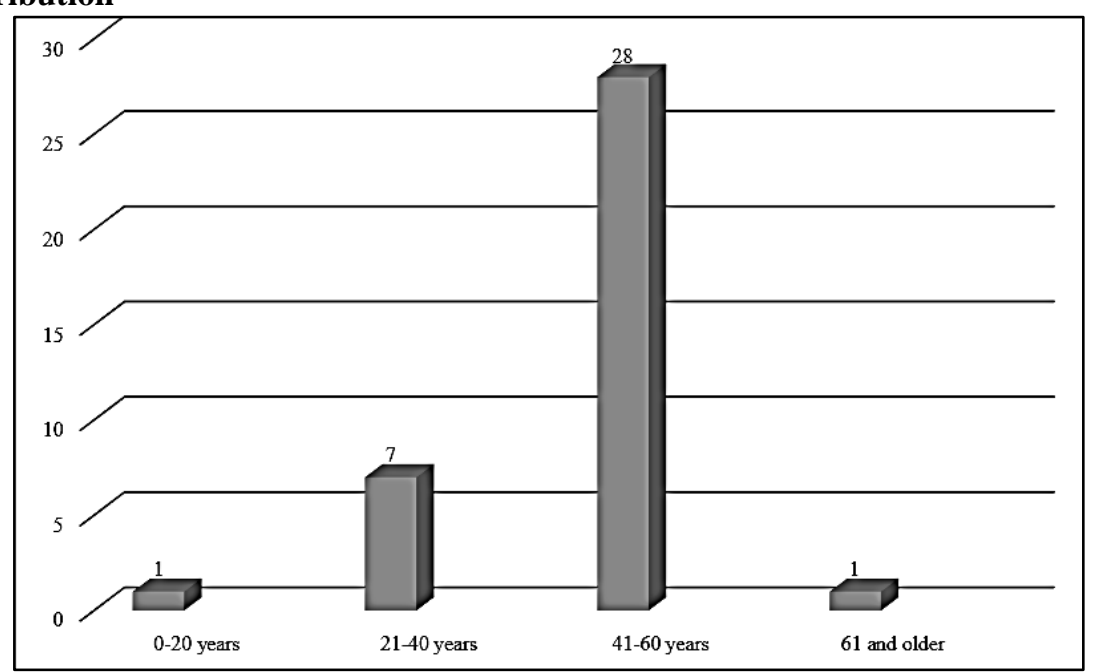

\section{Discussion}

Sarcoidosis is a chronic, immunologically mediated, multisystem disorder. ${ }^{1}$ The etiology is not clearly known, but various infectious agents including mycobacteria, herpes group of viruses and environmental factors have been implicated. ${ }^{5}$ The pathological characteristic of the disease is a granulomatous inflammation of various systems with the formation of non-caseating granulomas. ${ }^{3}$ The respiratory system is most commonly involved. Other systems involved include the skin, lymph nodes, salivary glands, heart, liver and nervous system. In our study we found the respiratory system is involved in almost $80 \%$ of patients, followed by ocular involvement in $38.9 \%$, dermatological involvement in $22.2 \%$, and neurological involvement in $11.1 \%$. Our prevalence rates are similar 
to those reported in the literature with regards to ocular, dermatological and nervous system involvement respectively. ${ }^{5,6}$

The diagnosis of sarcoidosis in a tuberculosisendemic country such as ours has been less owing to the overlap of clinical features between the two diseases. Clinicians preferred to err on the side of tuberculosis considering its wider prevalence and contagious nature. Moreover, facilities for biopsy and sample processing for the diagnosis of sarcoidosis were less. The condition was thought to be more prevalent in the socioeconomically affluent western countries. However, with improving standards of living, better control of tuberculosis, and largely due to improved diagnostic facilities, sarcoidosis is gaining recognition and being detected and treated at an earlier stage. ${ }^{7}$ Various studies have also described a differing presentation of this condition in our ethnicity, compared to the form seen in the Caucasians, Afro-Americans and Japanese. ${ }^{8}$

The ocular manifestations of sarcoidosis may be seen in $25-60 \%$ of the cases. The classical description of ocular involvement includes orbital inflammatory syndrome, lid and conjunctival granulomata, keratoconjunctivitis sicca, chronic granulomatous anterior uveitis, with large keratic precipitates, iris nodules and both anterior and posterior synechiae. Granulomata in the trabecular meshwork may also be present. Posterior segment involvement may present with intermediate uveitis with vitritis and snow balls in vitreous, multifocal choroiditis, retinal vasculitis with perivascular exudation classically described as candle wax drippings. Optic nerve head involvement may present as Opto ciliary shunts or granulomata. ${ }^{5}$ A report from India has mentioned a $29 \%$ ocular involvement which is very similar to our result. It also failed to notice the bimodal age distribution or female preponderance in ocular sarcoidosis, a contrast to western reports and found the prevalence of posterior uveitis to be more common in our population. $^{9}$

In our study, of the 22 patients who underwent an ophthalmological exam, 13 had uveitis, one had a third cranial nerve palsy, 6 had no features of ocular involvement and two had conditions which were probably unrelated to sarcoidosis.

Of the 13 with uveitis, keratic precipitates, vitritis, and posterior synechiae were seen in 11 patients; iris nodules in 10; retinal vasculitis in 4 and choroiditis in 3 patients. Our results indicate an increased anterior compared to posterior segment inflammation in our cohort compared to results by Khanna et al. ${ }^{9}$

Investigations in sarcoidosis include nonspecific indicators including serum angiotensin converting enzyme (s ACE) and lysozyme levels. ${ }^{10}$ Hilar lymphadenopathy detected by chest radiography or highresolution computed tomography (HRCT)scan is a more sensitive test. ${ }^{11}$ Specific confirmation, however, can be obtained only by detecting the characteristic granulomatous features in biopsied tissue. In respiratory or mediastinal lymphadenopathy, a biopsy may be obtained using bronchoalveolar lavage, or bronchoscopy-assisted specimen retrieval. Of late, endobronchial ultrasound guided transbronchial needle aspiration (EBUS-TBNA) of mediastinal lymph nodes with or without transbronchial lung biopsy (TBLB) has been found to have high diagnostic yield especially in early stages of pulmonary sarcoidosis. ${ }^{12}$ The dermatological involvement of sarcoidosis may present an ease of access to the biopsy site. However, as the lesions are difficult to diagnose owing to similarity to other conditions, a thorough examination with high index of suspicion is needed to arrive at a diagnosis. ${ }^{6}$ In our cohort of 36 sarcoidosis patients, Hilar lymphadenopathy was detected in 32 patients, sACE was elevated in 25 patients, skin biopsy in 3 patients and BAL cytology features in one patient.

The diagnosis of ocular sarcoidosis is further complicated by the fact that it may present without coexisting systemic involvement. As the focus of inflammation is small, most of the diagnostic features including hilar lymphadenopathy and elevated serum ACE levels may be absent. The presence of coexisting cutaneous or lid involvement in these cases may provide an opportunity for biopsy confirmed diagnosis. Another factor in the diagnosis of ocular sarcoidosis in endemic regions is the similarity of its clinical features with ocular tuberculosis. In the absence of coexisting systemic features, the ability to differentiate the two rests solely on certain specific investigative modalities. In such circumstances, the ruling out of Mycobacterium tuberculosis infection is preferred over investigating for sarcoidosis. Intraocular fluid analysis using real time Polymerase chain reaction (RT-PCR) can help in the identification of tuberculosis. ${ }^{13}$ The presence of a positive Mantoux test helps to rule out sarcoidosis, which is characterized by cutaneous anergy. The role of interferon gamma assays such as Quantiferon TB Gold, is less specific as there have been some reports of positivity even in sarcoidosis. The role of Mantoux test in the diagnosis of pulmonary tuberculosis in our country is limited due to its low specificity in the presence of high prevalence of latent tuberculosis. ${ }^{14}$ Hence it is not routinely asked in all patients with respiratory symptoms. In our study, of the 18 patients who had been administered the Mantoux test, all were negative, supporting the diagnosis of sarcoidosis.

To assist the diagnosis of ocular sarcoidosis, the international working group for ocular sarcoidosis (IWOS) framed guidelines integrating clinical features and investigations. ${ }^{4}$ Applying these guidelines in our patients with uveitis, we found, two had definite ocular sarcoidosis; seven had presumed ocular sarcoidosis; four had probable ocular sarcoidosis and none with possible ocular sarcoidosis.

As chronic uveitis, which is relatively painless, is one of the most frequent ocular manifestation of sarcoidosis, if not diagnosed in time, may result in sight 
threatening complications including band shaped keratopathy, complicated cataract, secondary glaucoma, cystoid macular edema, and retinal or optic atrophy. ${ }^{5}$ In our cohort of 36 patients with systemic sarcoidosis, only $22(61.1 \%)$ had undergone an ophthalmological evaluation after their diagnosis. Hence the awareness of ocular involvement should be communicated to other specialties dealing with sarcoidosis, so as to ensure a holistic approach in its management and better quality of life for the patient.

\section{Conclusion}

Sarcoidosis is not uncommon in India. Ocular features are common and need to be screened in all patients diagnosed with systemic sarcoidosis. An ophthalmologist can use IWOS criteria in cases of uveitis to clinically diagnose and refer cases for systemic evaluation of sarcoidosis.

Contributors: All the authors contributed to the design of the study. YSK and SA have contributed to the manuscript preparation.

\section{Funding: Nil}

Competing Interest: None declared

Ethics Approval: The study was approved by the Institutional Ethical committee.

\section{References}

1. Newman LS, Rose CS, Maier LA. Sarcoidosis. $N$ Engl J Med. 1997;336(17):1224-34.

2. James DG, Anderson R, Langley D, Ainslie D. Ocular Sarcoidosis. Br J Ophthalmol. 1964 Sep;48:461-70.

3. Babu K. Sarcoidosis in tuberculosis-endemic regions: India. J Ophthalmic Inflamm Infect. 2013 Jun 27;3(1):53.

4. Herbort CP, Rao NA, Mochizuki M; members of Scientific Committee of First International Workshop on Ocular Sarcoidosis. International criteria for the diagnosis of ocular sarcoidosis: results of the first International Workshop on Ocular Sarcoidosis (IWOS). Ocul Immunol Inflamm. 2009 May-Jun;17(3):160-9.

5. Rothova A. Ocular involvement in Sarcoidosis. $\mathrm{Br} J$ Ophthalmol 2000;84(1):110-116.

6. Fernandez-Faith E, McDonnell J. Cutaneous sarcoidosis: differential diagnosis. Clin Dermatol. 2007 MayJun;25(3):276-87.

7. Gupta D, Vinay N, Agarwal R, Agarwal AN. Sociodemographic profile of patients with sarcoidosis vis-à-vis tuberculosis. Sarcoidosis Vasc Diffuse Lung Dis. 2013 Nov 25;30(3):186-93.

8. Gupta SK. Sarcoidosis: a journey through 50 years. Indian J Chest Dis Allied Sci. 2002 Oct-Dec;44(4):24753.

9. Khanna A, Sidhu U, Bajwa G, Malhotra V. Pattern of ocular manifestations in patients with sarcoidosis in developing countries. Acta Ophthalmologica Scandinavica 2007;85(6):609-612.

10. Turton CW, Grundy E, Firth G, Mitchell D, Rigden BG, Turner-Warwick M. Value of measuring serum angiotensin I converting enzyme and serum lysozyme in the management of sarcoidosis. Thorax. 1979 Feb;34(1):57-62.

11. Kahkouee S, Samadi K, Alai A, Abedini A, Rezaiian L. Serum ACE Level in Sarcoidosis Patients with Typical and Atypical HRCT Manifestation. Pol J Radiol. 2016 Sep 23;81:458-461.

12. Hu LX, Chen RX, Huang H, Shao C, Wang P, Liu YZ, $\mathrm{Xu} \mathrm{ZJ}$. Endobronchial Ultrasound-guided Transbronchial Needle Aspiration versus Standard Bronchoscopic Modalities for Diagnosis of Sarcoidosis: A Meta-analysis. Chin Med J (Engl). 2016 Jul 5;129(13):1607-15.

13. Shetty SB, Biswas J, Murali S. Real-time and nested polymerase chain reaction in the diagnosis of multifocal serpiginoid choroiditis caused by Mycobacterium tuberculosis - a case report. J Ophthalmic Inflamm Infect. 2014 Dec;4(1):29.

14. Syed Ahamed Kabeer B, Raman B, Thomas A, et al. Role of QuantiFERON-TB gold, interferon gamma inducible protein-10 and tuberculin skin test in active tuberculosis diagnosis. PLoS One. 2010 Feb 4;5(2):e9051.

How to cite this article: Kamath, YS, Abbagani S. A study of ocular sarcoidosis using international workshop for ocular sarcoidosis criteria in a south Indian population. Ind J Clin Exp Ophthalmol. 2018;4(3):309-312. 